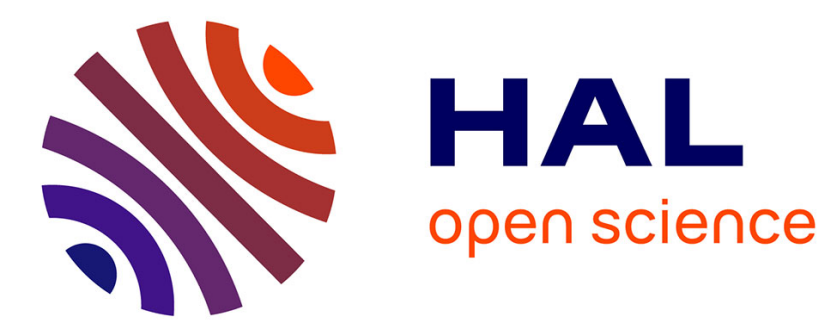

\title{
Dispositif bistable magnéto-optique
}

\author{
I.M. Popescu, A.G. Podoleanu, P.E. Sterian
}

\section{To cite this version:}

I.M. Popescu, A.G. Podoleanu, P.E. Sterian. Dispositif bistable magnéto-optique. Revue de Physique Appliquée, 1983, 18 (5), pp.313-315. 10.1051/rphysap:01983001805031300 . jpa-00245097

\section{HAL Id: jpa-00245097 https://hal.science/jpa-00245097}

Submitted on 1 Jan 1983

HAL is a multi-disciplinary open access archive for the deposit and dissemination of scientific research documents, whether they are published or not. The documents may come from teaching and research institutions in France or abroad, or from public or private research centers.
L'archive ouverte pluridisciplinaire HAL, est destinée au dépôt et à la diffusion de documents scientifiques de niveau recherche, publiés ou non, émanant des établissements d'enseignement et de recherche français ou étrangers, des laboratoires publics ou privés. 


\title{
Dispositif bistable magnéto-optique
}

\author{
I. M. Popescu, A. G. Podoleanu et P. E. Sterian \\ Laboratoire d'Opto-électronique, Département de physique, Institut Polytechnique de Bucarest, Roumanie
}

(Reçu le 4 octobre 1982, révisé le 11 février 1983, accepté le 21 février 1983)

Résumé. - L'article présente un dispositif mixte bistable magnéto-optique, réalisé à l'aide d'une cavité laser active à laquelle est appliqué un champ magnétique axial, le champ étant contrôlé par une boucle de contre-réaction dans le dispositif. Abstract. - In this paper we present a mixed magneto-optical bistable device, realized to the active cavity of a
laser by applying an axial magnetic field, the field being driven electronically by means of a feedback loop.

1. Introduction. - Les phénomènes de bistabilité optique et électro-optique ont été étudiés théoriquement et expérimentalement et ont conduit à l'élaboration d'une large gamme de dispositifs à applications pratiques [1-4].

Dans ce travail est présenté un nouveau type de dispositif " magnéto-optique " dont le comportement bistable est déterminé par la commande adéquate d'un champ magnétique axial, appliqué dans la cavité laser et contrôlé par une boucle de contreréaction dans le dispositif.

2. Description de la configuration expérimentale. La configuration expérimentale du dispositif est présentée dans le schéma bloc de la figure 1 .

Le laser utilisé est un laser $\mathrm{HeNe}$ multimode travaillant sur la longueur d'onde $\lambda=0,6328 \mu \mathrm{m}$ avec une puissance de $1 \mathrm{~mW}$, et dont les miroirs confocaux sont distants de $75 \mathrm{~cm}$.

Le tube laser, équipé de fenêtres de Brewster et de diamètre $3 \mathrm{~mm}$ est excité par un courant continu de $10 \mathrm{~mA}$, la longueur de décharge ayant $65 \mathrm{~cm}$.

Autour du tube laser, dans une position symétrique par rapport à ses extrémités, est placée une bobine en fil de cuivre de $0,5 \mathrm{~mm}$ de diamètre et dont le support a un diamètre de $2 \mathrm{~cm}$ pour une longueur de $8 \mathrm{~cm}$.

Le champ magnétique axial produit par la bobine est de $6 \times 10^{-4} \mathrm{~T} / \mathrm{A}$ environ.

Le courant $i$ dans la bobine est obtenu par amplification du signal délivré par le photodétecteur FD à l'aide d'un circuit intégré ROB 733 suivi à un étage de puissance EP.

Le faisceau optique $I_{\mathrm{d}}$ qui tombe sur le photodétecteur est la superposition réalisée dans l'étage $\Sigma$, de deux composantes : une composante cohérente

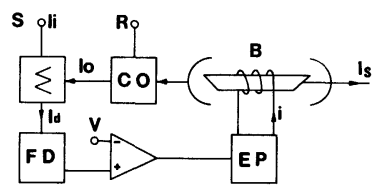

Fig. 1. - Schéma-bloc du dispositif bistable magnétooptique ; $\Sigma$ : étage d'addition optique ; FD : photodétecteur ; EP : étage de puissance ; $B$ : bobine entourant le tube laser; $V$ : tension de référence; $\mathrm{CO}:$ commutateur optique; $I_{\mathrm{i}}$ : signal optique d'entrée; $I_{0}:$ signal de réaction $; I_{\mathrm{d}}$ : signal sur les photodétecteurs; $I_{\mathrm{s}}:$ signal optique de sortie du dispositif ; S, R : entrées pour le déclenchement (signal optique) et la coupure (signal de tension) du cycle.

[Block diagram of the bistable magneto-optical device; $\Sigma$ : addition stage ; FD : photodetector ; EP : power stage ; B : coil around the laser tube; $V:$ reference voltage; CO : optical switch; $I_{\mathrm{i}}$ : input optical signal $; I_{0}$ : feedback signal ; $I_{\mathrm{d}}$ : signal on the photodevice; $I_{\mathrm{s}}$ : optical output signal; $\mathrm{S}, \mathrm{R}$ : set and reset inputs.]

notée $I_{0}$, produite par le laser et qui traverse le commutateur optique $\mathrm{CO}$, et une composante notée $I_{\mathrm{i}}$, cohérente ou incohérente, amenée à $\Sigma$ à partir d'une source extérieure.

Comme commutateur optique on a utilisé une valve à cristal liquide ou un modulateur électrooptique d'intensité.

3. Fonctionnement bistable du dispositif. - A l'aide de ce dispositif nous avons étudié l'effet du champ magnétique axial sur l'intensité de l'oscillation laser. Les résultats obtenus sont conformes à ceux donnés dans la littérature [5-8].

Modifiant le courant $i$ dans la bobine dans une 
plage comprise entre 0 et $4 \mathrm{~A}$ nous avons obtenu une dépendance approximativement linéaire entre l'intensité du signal laser $I_{0}$ et le courant $i$ pour différentes valeurs des pertes dans la cavité laser (Fig. 2).

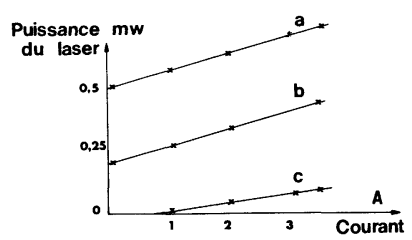

.Fig. 2. - Variation de la puissance laser $I_{0}$ en fonction du courant $i$ dans la bobine pour différentes valeurs des pertes de la cavité. Le coefficient de pertes $\delta$ augmente de la courbe $a$ ) à la courbe $c$ ).

[Output power of the laser versus coil current for different losses of the cavity. The losses increase from curve $a$ ) to curve $c$ ).]

Le coefficient de pertes $\delta$ a été modifié en jouant sur la position des miroirs de la cavité laser. Ce coefficient est minimum pour la courbe notée $a$ ) dans la figure 2 .

Les éléments de la boucle de réaction de la structure mixte électro-optique présentée dans la figure 1 sont connectés de façon à assurer une contre-réaction positive vis-à-vis du signal $I_{0}$ fourni par le laser.

Le signal de sortie $I_{\mathrm{s}}$ de ce dispositif à laser, étant proportionnel au signal $I_{0}$, a alors deux états stables stationnaires.

Les deux niveaux « haut » et « bas » du signal laser utilisé dans la boucle de contre-réaction, respectivement $I_{0}^{+}$et $I_{0}^{-}$, qui caractérisent le comportement bistable du dispositif, sont situés dans la région quasilinéaire du diagramme qui exprime la dépendance de l'intensité laser en fonction du courant de la bobine B (cf. Fig. 2) [5, 8].

La chaîne d'amplification électronique détermine par ces non-linéarités les valeurs $I_{0}^{-}$et $I_{0}^{+}$du signal $\mathrm{du}$ laser, auxquelles correspondent pour le signal optique arrivant à l'entrée du photodétecteur respectivement deux valeurs $I_{\mathrm{d}}^{+}$et $I_{\mathrm{d}_{-}^{-}}^{-}$.

Lorsque le signal d'entrée sur la porte $S$ est tel que $I_{\mathrm{i}}>I_{\mathrm{i}}^{+}$, où $I_{\mathrm{i}}^{+}=I_{\mathrm{d}}^{+}-I_{0}^{-}$, le dispositif bascule dans l'état supérieur. Cet état est caractérisé par une intensité $I_{0}^{+}$qui correspond à la limite supérieure de la gåmme dynamique de l'amplificateur.

Ceci explique l'indépendance du signal laser $I_{0}$ par rapport à un accroissement du signal d'entrée $I_{\mathrm{i}}$ au-dessus de la valeur $I_{i}^{+}$.

Le retour du dispositif à l'état initial est obtenu par diminution du signal d'entrée $I_{\mathrm{i}}$ au-dessous de la valeur limite $I_{\mathrm{i}}^{-}$, définie par : $I_{\mathrm{i}}^{-}=I_{\mathrm{d}}^{-}-I_{0}^{+}$.

Les paramètres caractéristiques du cycle d'hystérésis du dispositif sont indiqués dans la figure 3.

La courbe d'hystérésis visualisée sur un tube cathodique est présentée dans la figure 4. Sur les axes sont

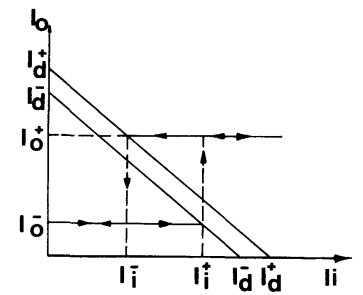

Fig. 3. - Cycle d'hystérésis du dispositif; $I_{0}$ : signal optique laser utilisé dans la voie de réaction; $I_{\mathrm{i}}$ : signal optique d'entrée; $I_{0}^{-}, I_{0}^{+}$: valeurs des deux niveaux stables émis par le laser $; I_{\mathrm{i}}^{+}$: valeur seuil de $I_{\mathrm{i}}$ pour laquelle le dispositif commute dans l'état supérieur; $I_{\mathrm{i}}^{-}$: valeur seuil pour laquelle le dispositif commute dans l'état inférieur; $I_{\mathrm{d}}^{-}$et $I_{\mathrm{d}}^{+}$sont définis dans le texte.

[Hysteresis cycle of the device; $I_{\mathrm{i}}$ : input optical signal; $I_{0}$ : optical signal from the laser used in the feedback loop; $I_{0}^{-}, I_{0}^{+}$: the two stables emission levels of the laser; $I_{\mathrm{i}}^{+}$: the threshold to bascule in the upper state $; I_{\mathrm{i}}^{-}$: the threshold to bascule in the lower state; $I_{\mathrm{d}}^{-}$and $I_{\mathrm{d}}^{+}$are defined in the text.]

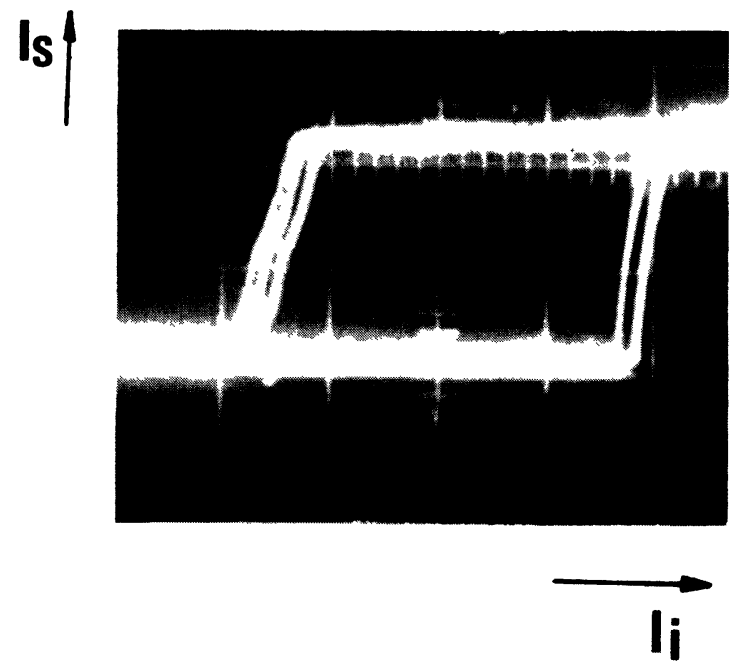

Fig. 4. - Oscillogramme du cycle d'hystérésis du dispositif.

[Oscillogram of the hysteresis cycle of the device.]

représentées les grandeurs définies dans la figure 3 avec la remarque que $I_{\mathrm{s}}$ est proportionnel aux $I_{0}$. Pour certaines valeurs de la tension de référence $V$ de l'amplificateur et du coefficient de pertes $\delta$ on obtient la condition $I_{0}^{+}>I_{\mathrm{d}}^{-}$; aussi faut-il couper la voie de réaction pour assurer le retour du dispositif.

Cette coupure est réalisée à l'aide d'une impulsion de tension appliquée sur la porte $\mathrm{R}$ du commutateur optique placé sur le faisceau laser $I_{0}$. La largeur et la position du cycle d'hystérésis sont influencées par les paramètres $\delta, V$ et $I_{0}$.

Si on modifie ces paramètres, on obtient des comportements différents du système : comme dispositif 
bistable SR à deux entrées, ou comme formateur des deux niveaux optiques (trigger Schmitt).

4. Conclusions. - Nous avons démontré la possibilité de réaliser un dispositif bistable mixte magnétooptique qui permet d'obtenir en sortie un signal optique cohérent $I_{\mathrm{s}}$ pouvant prendre deux valeurs stables.

Le principe de ce type de dispositif utilise la théorie des lasers Zeeman et la variation de l'intensité de sortie du laser en fonction de l'intensité du champ magnétique axial.

Le cycle hystérésis du dispositif décrit dépend des paramètres $\delta, V$ et $I_{0}$, définis dans l'article.

En modifiant ces paramètres on peut changer la largeur et la position du cycle d'hystérésis et obtenir ainsi des comportements différents du dispositif en tant qu'élément de circuit magnéto-optique.

\section{Bibliographie}

[1] McCall, S. L., Phys. Rev. 188 (1974) 1515.

[2] Bonifacio, R., Lugiato, L. A., Opt. Commun. 19 (1976) 172.

[3] Gibbs, H. M., McCall, S. L. and Venkatesan, T. N. C., Phys. Rev. Lett. 36 (1976) 1135.

[4] Smith, P. W. and Turner, E. H., Appl. Phys. Lett. 30 (1977) 280.
[5] Culshaw, W. and Kannellaud, J., Phys. Rev. A 133 (1964) 691.

[6] Kannellaud, J. and Culshaw, W., Phys. Rev. 141 (1966) 228.

[7] Herr, C. W. and Graft, R. D., Phys. Rev. A 140 (1965) 1088.

[8] Baer, T., Kowalski, F. V. and Hall, J. L., Appl. Opt. 19 (1980) 3173. 